\title{
THE JOVIAN HYDROGEN BULGE: EVIDENCE FOR CO-ROTATING MAGNETOSPHERIC CONVECTION
}

\author{
A. J. DESSLER \\ Department of Space Physics and Astronomy, Rice University, Houston, TX 77001 U.S.A. \\ B. R. SANDEL \\ Earth and Space Sciences Institute, University of Southern California, Tucson, AZ 85713 U.S.A. \\ and \\ S. K. ATREYA \\ Department of Atmospheric and Oceanic Science, University of Michigan, Ann Arbor, MI 48109 U.S.A.
}

(Received 29 August 1980)

\begin{abstract}
The hydrogen bulge is a feature in Jupiter's upper atmosphere that co-rotates with the planetary magnetic field (i.e. the hydrogen bulge is fixed in System III coordinates). It is located approximately $180^{\circ}$ removed in System III longitude from the active sector, which has been identified as the source region for Jovian decametric radio emission and for release of energetic electrons into interplanetary space. According to the magnetic-anomaly model, the active sector is produced by the effect of the large magnetic anomaly in Jupiter's northern hemisphere. On the basis of the magneticanomaly model, it has been theoretically expected for some time that a two-cell magnetospheric convection pattern exists within the Jovian magnetosphere. Because the convection pattern is established by magnetic-anomaly effects of the active sector, the pattern co-rotates with Jupiter. (This is in contrast to the Earth's two-cell convection pattern that is fixed relative to the Sun with the Earth rotating beneath it.) The sense of the convection is to bring hot magnetospheric plasma into the upper atmosphere in the longitude region of the hydrogen bulge. This hot plasma contains electrons with energies of the order of $100 \mathrm{keV}$ that dissociate atmospheric molecules to produce the atomic hydrogen that creates the observed longitudinal asymmetry in hydrogen Lyman alpha emission. We regard the existence of the hydrogen bulge as the best evidence available thus far for the reality of the expected co-rotating magnetospheric convection pattern.
\end{abstract}

\section{INTRODUCTION}

A pronounced longitudinal asymmetry in the midlatitude H Lyman alpha ( $L y \alpha$ ) brightness of Jupiter has been inferred by Sandel et al. (1980) using data from the Voyager Ultraviolet Spectrometer (UVS). The UVS instruments are objective grating spectrometers covering the wavelength range of approx. $500-1700 \AA$ with 126 contiguous photon-counting channels. The instrument and its calibration and data reduction procedures have been described by Broadfoot et al. (1977) and Broadfoot et al. (1980).

The observed Ly $\alpha$ asymmetry has been interpreted by Sandel et al. (1980), as being caused by a corresponding longitudinal asymmetry in the atomic hydrogen column content of Jupiter's upper atmosphere. The observed $L y \alpha$ bulge and the inferred atomic hydrogen bulge are directly correlated because $L y \alpha$ airglow from Jupiter is produced primarily by resonance scattering of solar Lya photons by $\mathbf{H}$. The asymmetry appears to be fixed in System III coordinates, so the hydrogen bulge co-rotates with Jupiter's magnetic field. Sandel et al. (1980) and Clarke et al. (1980) propose that the extra hydrogen within the bulge is produced by electron bombardment of Jupiter's atmosphere. (No suggestion has, to our knowledge, been made for any internal, upward-propagating mechansim for producing a hydrogen bulge; moreover, it is difficult to imagine an internal source fixed to the magnetic coordinate system.) The magnetospheric problem posed by the hydrogen bulge in this view thus reduces to finding the mechanism that produces a longitudinal asymmetry in electron flux bombarding Jupiter's upper atmosphere with an energy spectrum adequate to create the required source flux of atomic hydrogen.

The magnetic-anomaly model has been applied to predict or explain a wide variety of Jovian magnetospheric phenomena (see Dessler and Vasyliunas, 1979; Vasyliunas and Dessler, 1980, and references contained therein). In this paper we show that the electron flux asymmetry is a natural 
consequence of the expected large-scale magnetospheric convection pattern that, because of magnetic-anomaly effects, co-rotates with Jupiter (Vasyliunas, 1978; Hill, 1980a; Hill et al., 1980). We therefore conclude that the hydrogen bulge is direct evidence of the existence of co-rotating magnetospheric convection.

\section{THE H LYMAN a ASYMMETRY}

Here we extend the analysis presented by Sandel et al. (1980) to include the north-south structure in $L y \alpha$ intensity by plotting $L y \alpha$ isophote maps and drawing on data from special high-resolution northsouth scans. Isophote maps have been generated using data from the pre-encounter North-South Map sequence on both spacecraft. The observing sequence, as shown in Fig. 1a, consisted of a series of swaths from north pole to south pole, approximately along the central meridian of the planet. Each swath was made up of either 13 or 16 steps in latitude with each step having a duration of $48 \mathrm{~s}$. Swaths of 13 and 16 steps were repeated alternately at intervals of about $12 \mathrm{mins}$, or $7^{\circ}$ in rotation phase, during one rotation period of the planet. Jupiter was therefore mapped in latitude (by stepping the scan platform) and longitude (by taking advantage of the planet's rotation).

The orientation of the UVS slit during the N-S Map sequence was not well suited for studying N-S structure because its long dimension extended approximately north-south, reducing spatial resolution in that direction. Fortunately the VOYAGER 1 "Slitscan" sequence yielded data at much higher north-south resolution as shown in Fig. 1b. This favorable slit orientation was available for only a short time because of the characteristics of the trajectory, so longitude coverage was limited to two scans separated by about $17^{\circ}$. Each of these two scans moved the UVS slit from pole to pole in about $12 \mathrm{~min}$.

For both sequences, the true $L y \alpha$ signal was derived by subtracting a background consisting of $\mathrm{H}_{2}$ Lyman and Werner band emissions excited in the atmosphere and of noise induced by highenergy particles. Since the entire spectral range was detected continuously, this background was determined from portions of the spectrum not containing Ly $\alpha$ signal. For the N-S Maps, the background amounted to about $15 \%$ of the $L y \alpha$ signal. The Slitscan sequence was executed much closer to the planet in a more severe radiation environment, and the noise rate was about $60 \%$ of the true $L y \alpha-$ induced count rate. The error bars in the figures include counting statistics in the signal and in the
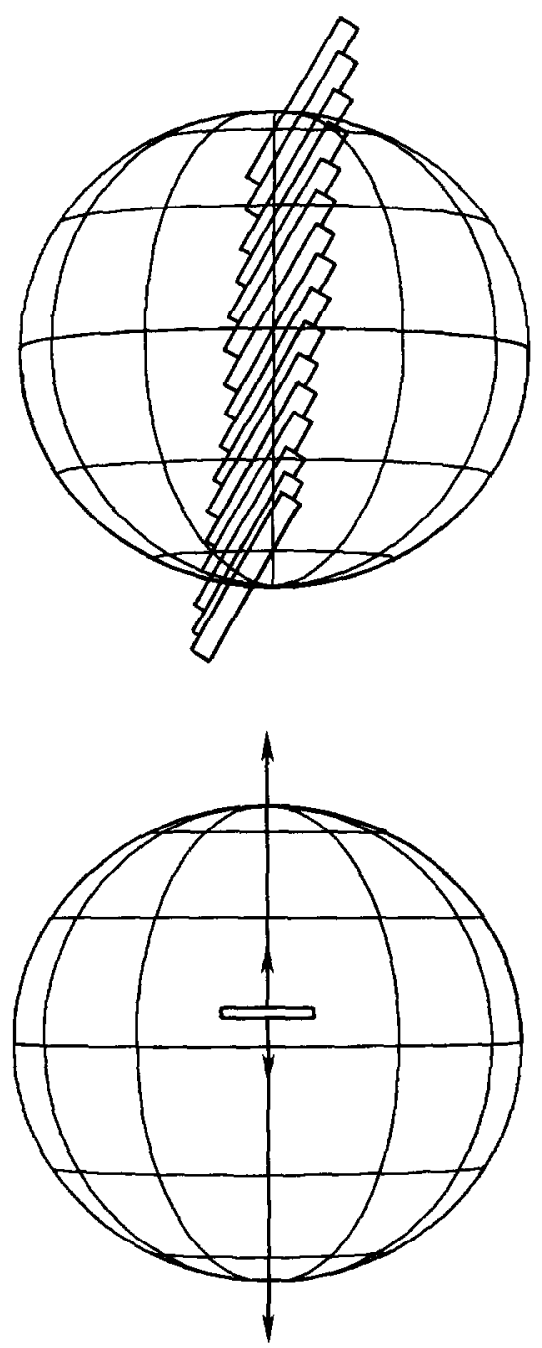

Fig. 1. OBSERVING GeOMETRY, SHOWING THE $0.1^{\circ} \times$ $0.86^{\circ}$ UVS SLIT PROJECTED ON THE PLANET.

(a) NokTH-SOUih MaP SEquence.

The rectangles show the slit positions for each of the 16 steps of a N-S Map swath. The range from the planet was $46 R$.

(b) SLITSCAN SEQUENCE.

The slit was scanned slowly from south pole to north pole and back, giving the best available $N-S$ resolution at mid-latitudes. The range was $25 R_{J}$.

background subtraction process; they represent the $\pm 1 \sigma$ uncertainty of the data points.

The Ly $\alpha$ isophote maps derived from the N-S Map sequences of the two spacecraft are shown in Fig. 2a (taken from Broadfoot et al., 1980) and Fig. 2b. Each tick mark along the longitude scale corresponds to one north-south swath. The sawtooth variation that is particularly noticeable near the 

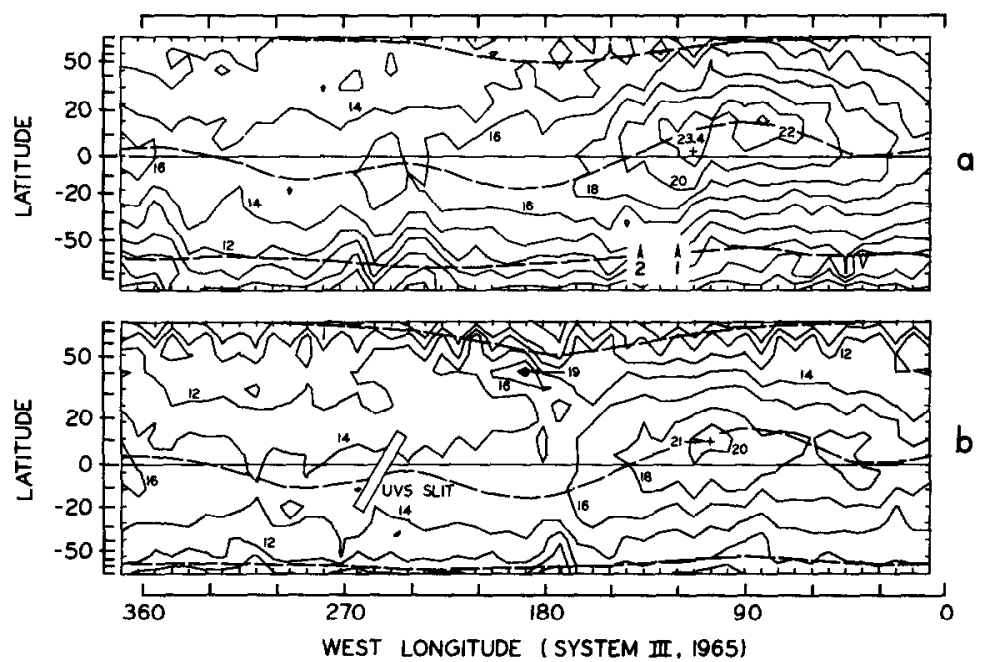

Fig. 2. LYMAN $\alpha$ ISOPHOTE MAPS OF JUPIER MADE BY VOYAGER 1(a) AND VOYAGER 2(b).

The units are kiloRayleighs, and the contour interval is $2 \mathrm{kR}$. The rectangle in (b) shows the size and orientation of the UVS slit. The coordinate scales refer to the center of the slit. The longitudes of scan 1 and scan 2 of the Slitscan sequence are indicated by the numbered arrowheads in (a). Also shown (by dashed lines) are the projections of the foot of the Io flux tube and the particle drift equator. Clearly defined near $100^{\circ} \mathrm{W}$, the $L y \alpha$ intensity bulge is displaced to the north of the spin equator, but it is roughly coincident with the particle drift equator.

north pole in Fig. 2b is not real. It results from the fact that alternate swaths of 13 steps have been processed to fit into the 16-step grid established by the other swaths. Slight inaccuracies in position occur in fitting the two scan designs together, leading to the sawtooth pattern. The latitude scales of the two maps differ because of slightly different sequence designs and because the two spacceraft were at different latitudes when the observations were made.

The $L y \alpha$ intensity bulge is clearly apparent in both maps, and the earlier analysis, which showed that the longitude of the bulge was the same at both encounters, is confirmed. As before, the intensity measured by VOYAGER 1 is somewhat higher than that measured by VOYAGER 2. Revealed for the first time by this extended analysis is the fact that the bulge shows a pronounced north-south asymmetry in Jovigraphic coordinates. Although the brightest region is $8^{\circ}-12^{\circ}$ above the spin equator in both maps, this region is centered about the particle drift equator. (The particle drift equator and its significance to magnetospheric convection are discussed in Section 3). The coincidence between the $L y \alpha$ intensity bulge and the particle drift equator is clearly seen in Fig. 3. The data at mid-latitudes were taken with the slit well away from the bright auroral regions near the poles, so the isophotes could not have been distorted by auroral contamination. The brightnesses shown are averages over the slit, whose north-south extent is considerable as illustrated by the slit projection in Figs. 1a and $2 \mathrm{~b}$. This means that the true northsouth peak in equatorial brightness could have sharper features than shown by the isophotes because a sharp peak would be smeared by integration over the length of the slit. The true brightness profile can in principle be derived by deconvolving the slit length response from the data. However, because of the limitations imposed by the small number of data points in each swath (13 or 16) and the complex structure in the $L y \alpha$ brightness near the poles, such a deconvolution is impractical. Fortunately the information that would be derived from deconvolution is available from another source, the Slitscan sequence discussed later.

The data that make up the bright spot near latitude $40^{\circ} \mathrm{N}$, longitude $190^{\circ} \mathrm{W}$ in the VOYAGER 2 map (Fig. 2b) have withstood scrutiny, and we believe the feature is real. One possible explanation for such a spot could be electron bombardment associated with the foot of the Io (or Europa) flux tube (Goldreich and Lynden-Bell, 1969; Atreya et al., 1977; Dessler and Chamberlain, 1979). Because of the north-south extent of the slit, the bright spot's appearance when the center of the slit was at $40^{\circ} \mathrm{N}$ is consistent with the bright region being located on field lines that thread the torus. 
This can be illustrated by imagining the field of view in Fig. $2 b$ being translated without rotation so that it is centered on the bright spot. Then the path of the foot of Io's flux tube lies with the field of view, and a bright spot on the path could be detected. A small bright spot could have fallen outside the UVS slit in the more northerly slit positions since the slit was tilted relative to its motion. However, although the northern foot of Io's flux tube was visible from the spacecraft at this time, it was near the east limb of the planet, far from the UVS field-of-view at the time the bright spot was detected. The foot of Europa's flux tube was behind the planet. [Flux tube foot positions were kindly supplied by N. F. Ness (personal communication). They are based on the GSFC $\mathrm{O}_{4}$ magnetic-field model, 3 February 1979 Version.]

A more likely explanation is that this spot is a transient manifestation of the interaction between the active sector and the Io torus. Dessler (1980) has argued that, because of the observed gross longitudinal asymmetry in the mass density of the Io plasma torus (Trauger et al., 1980; Trafton, 1980; Pilcher and Morgan, 1980) a persistent, longitudinally-asymmetric pattern of Birkeland (magnetically-field-aligned) currents flow between the Io torus and the Jovian ionosphere. The density of the Birkeland current is a linear function of the longitudinal gradient in the mass density of the torus (Dessler, 1980); if the longitudinal density gradient is great enough, the current density would reach a critical value for which a current-driven plasma instability would develop (Smith and Goertz, 1978; Dessler and Hill, 1979) and accelerate particles into the atmosphere, thus creating a localized hot spot such as the one shown in Fig. $2 b$ at $\lambda_{\mathrm{III}}=190^{\circ}$. Its latitude and longitude are about as expected, and its transient nature (visible during the VOYAGER 2 flyby, but not during that of VOYAGER 1) is consistent with the ground-based observations of changes in longitudinal density gradients that would produce corresponding changes in localized Birkeland current densities. The origin of this unusual feature is not of central importance to the principal conclusion of this paper regarding co-rotating convection.

Additional data at high north-south resolution from the VOYAGER 1 Slitscan sequence described above confirm the existence of a northsouth asymmetry in the $L y_{\alpha}$ brightness. The longitudes of the Slitscans, shown in Fig. 2a, are near the longitude of peak brightness in the Lya bulge. The $L y \alpha$ intensities measured in the two scans are shown in Fig. 4. The peak brightness in Scan 1,

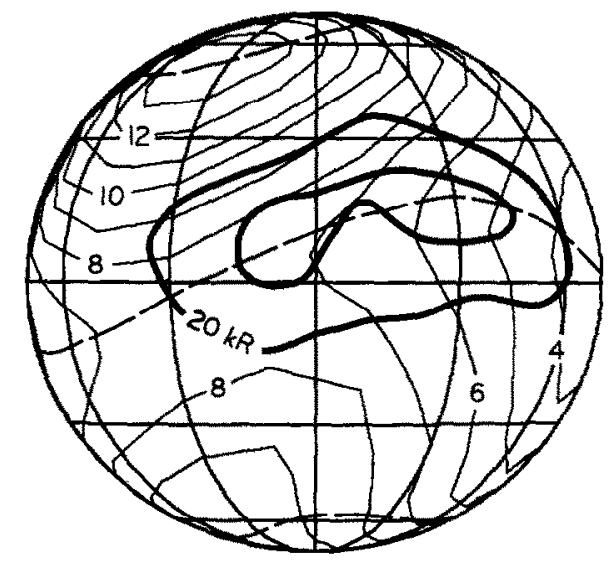

Fig. 3. Perspective view from a postrion $50 R_{j}$ DISTant FROM JUPTTER ILLUSTRATING THE RELATIONSHIP BETWEEN MAGNETIC FIELD CONTOURS AND THE HYDROGEN BULGE.

The view is from directly above the spin equator and a central meridian longitude $\lambda_{\text {III }}(1965)=110^{\circ}$. The light contour lines show the magnetic field magnitude in gauss. The hydrogen bulge is shown by the two heavy contours, which are the 20 and $22 \mathrm{kR}$ contours taken from Fig. 2a and smoothed by hand. The dashed lines show the projections of the feet of the lo flux tube and the particle drift equator (after Acuña and Ness, 1976).

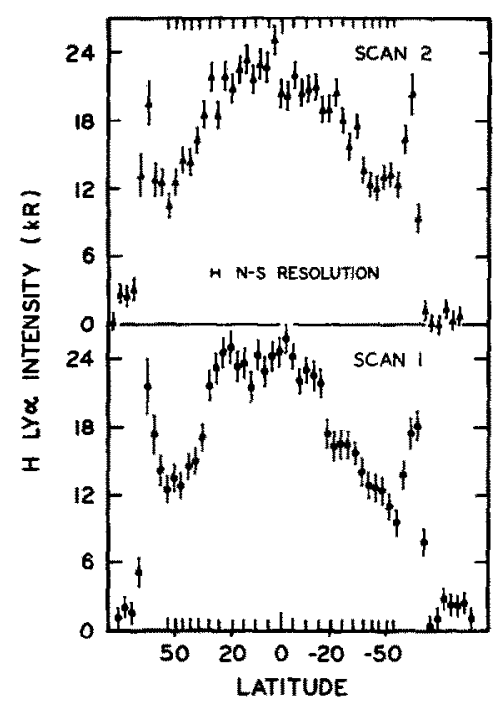

FIG. 4. Ly $\alpha$ INTENSITY VS LATTIUDE FROM THE TWO SCANS OF THE SLITSCAN SEQUENCE.

The latitude scale is reliable at mid-latitudes, but becomes less so within $30^{\circ}$ of the poles. The N-S resolution implied by the slit width is shown, and the error bars represent \pm one standard deviation statistical uncertainty. 
taken at smaller System III longitudes than Scan 2, is the higher as would be expected on the basis of Fig. 2a. In both scans the brightness peak occurs to the north of the spin equator and on the particle drift equator. The peak is broad, extending over $30^{\circ}-40^{\circ}$ in latitude. The shape resembles the isophote contours, so we conclude that little spatial information was lost at mid-latitudes by smearing the brightness over the length of the slit.

To compare the Slitscan to the N-S Map, we have smeared the data of Fig. 4 to the $\mathrm{N}-\mathrm{S}$ resolution implied by the slit length during the N-S Map Sequence. This procedure defines more clearly the maximum brightness averaged over the latitude coverage of the slit at the time of the N-S Map, about $35^{\circ}$ at the equator. For scan 1 the maximum brightness is $23.8 \mathrm{kR}$; for scan 2 it is $21.9 \mathrm{kR}$. The result of smearing is shown in Fig. 5, along with the intensity profile measured in the single swath of the N-S Map nearest the same longitude. Only scan 1 is shown here, but it is representative of scan 2 as well. According to the Slitscan data the maximum intensity at $120^{\circ} \mathrm{W}$ occurs $5^{\circ}-10^{\circ}$ north of the spin equator, somewhat closer to the equator than at the center of the bulge near $100^{\circ} \mathrm{W}$. This is consistent with Fig. 2, which shows that the isophotes are more symmetric about the spin equator at $120^{\circ} \mathrm{W}$ than at $100^{\circ} \mathrm{W}$, i.e. the spin equator and the particle drift equator are closer together at $120^{\circ}$ than $100^{\circ}$. (They, in fact, cross at about $\lambda_{\mathrm{III}}=140^{\circ}$.) The difference between the smoothed Slitscan data and the N-S Map data at high latitudes may be due to

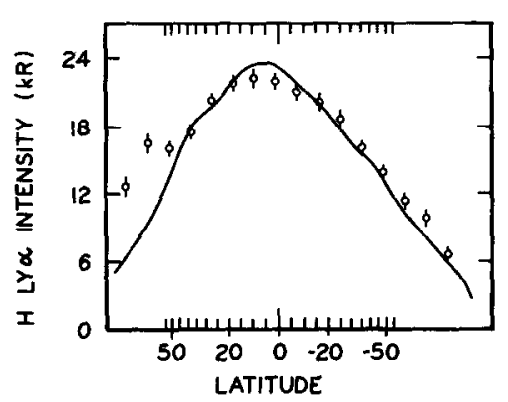

Fig. 5. THE DATA POINTS ARE THE Ly $\alpha$ INTENSTTY MEASURED DURING THE N-S MAP SWATH TAKFN AT THE LONGITUDE OF SCAN 1 AS SHOWN IN FIG. 2a.

For comparison with these data, the solid curve was derived by smearing the Slitscan data from scan 1 to the resolution of the N-S Map. The agreement in the positions of the peaks in both curves further confirms the displacement of the center of the $L y \alpha$ bulge northward from the spin equator to the particle drift equator. the fact that the smearing was done by assuming that the slit was aligned with the central meridian and moved along it, neglecting the true tilt and offset from the center of the planet. The difference could also be the result of a brighter northern aurora at the time of the N-S Map. In any case, the excellent agreement at mid-latitudes confirms the general $\mathrm{N}-\mathrm{S}$ distribution of intensity inferred from the N-S Map sequences.

A similar bulge in $L y \alpha$ has been inferred by Clarke et al. (1980) using data from an imaging spectrometer on board a sounding rocket. Their observation, made approximately three months before the VOYAGER 1 encounter, shows the bulge to be near $\lambda_{\text {III }}=80^{\circ}$, about $20^{\circ}$ eastward of the position determined here. This difference led Clarke et al. (1980) to suggest that the bulge may drift in longitude. Figure $2 a$ and $b$ may be consistent with an increase in the System III longitude of the bulge between the two VOYAGER encounters, which were separated by four months. As we will see in Section 3, such motions are consistent with observed changes in the Io torus, which would produce, through changes in the co-rotating convection pattern, apparent longitudinal motion of the bulge as well as changes in intensity.

Using a model of resonance scattering by the atmosphere that fits data outside the bulge reasonably well, Clarke et al. (1980) deduce an enhancement of $12 \mathrm{kR}$ in the bulge above the "normal" subsolar brightness of $18 \mathrm{kR}$. This enhancement by about $67 \%$ compares fairly well with the $56 \%$ enhancement inferred from Fig. $2 \mathrm{a}(15 \mathrm{kR}$ and $23.4 \mathrm{kR}$ in non-bulge and bulge regions, respectively) and the $50 \%$ enhancement inferred from Fig. $2 b$ (14 and $21 \mathrm{kR}$ ). It is therefore probable that the two experiments have measured the same phenomenon, and the Lyo bulge is established as a long-lived feature of Jupiter's atmosphere.

The absence of a comparable longitudinal variation in the $\mathrm{H}_{2}$ Iyman and Werner band emissions, which are excited by electrons, means that the excess $L y \alpha$ from the bulge is probably not produced by direct electron excitation. Rather, it is almost certainly the result of increased resonance scattering of solar radiation by a varying column density of atomic hydrogen above an absorbing hydrocarbon layer (see Sandel et al., 1980; Clarke et al., 1980). The required increase is from the non-bulge column density of $\mathrm{N}_{\mathrm{H}} \approx 1 \times 10^{17} \mathrm{~cm}^{-2}$ to $\mathrm{N}_{\mathrm{H}} \approx 3 \times 10^{17} \mathrm{~cm}^{-2}$ in the bulge region (McConnell et al., 1980a). An additional constraint is imposed by the detection of a longitudinal variation in He $584 \AA$ emission that is roughly $180^{\circ}$ out of 
phase with the Lya intensity (McConnell et al., 1980a). A number of self-consistent scenarios accounting for the longitudinal structure in both the Ly $\alpha$ and $\mathrm{He} 584 \AA$ emissions from Jupiter have been advanced by McConnell et al. (1980a), but the most likely are based on longitudinal asymmetries in particle precipitation patterns in either hard or soft electrons as originally suggested by Sandel et al. (1980). Furthermore, McConnell et al. (1980b), using observations of Jupiter's $L y \alpha$ nightglow, infer the presence of particle precipitation on the nightside and derive evidence for a longitudinal asymmetry in fluxes. They argue that the particles responsible for the nightside excitation probably have a soft energy spectrum.

Electrons soft enough to heat the atmosphere without exciting airglow emissions appear to offer one possibility for increasing the $\mathrm{H}$ column abundance; heating of the exosphere alone, even by large amounts, will not increase the $L y \alpha$ albedo significantly (Yung and Strobel, 1980). However, the $\mathrm{H}_{2}$ density profile, as measured by the $\alpha$ Len stellar occultation experiment (Festou et al., 1980), leads us to conclude that the energy of the electrons cannot be very small. This is because lowenergy electrons can dissociate $\mathrm{H}_{2}$ only high in the atmosphere where the $\mathrm{H}_{2}$ density is low and insufficient atomic hydrogen would be generated.

To produce enough atomic hydrogen, electrons must have energies approaching $100 \mathrm{keV}$. These electrons penetrate deeply and produce most of their secondary electrons low in the atmosphere where $\mathrm{H}_{2}$ band emission excited by the secondaries is absorbed by overlying hydrocarbons (Strobel, 1979). Formation of atomic hydrogen by dissociation of $\mathrm{H}_{2}$ and $\mathrm{CH}_{4}$ and strong heating of the atmosphere cause the $\mathrm{H}$ column abundance above the homopause to be increased. The required energy flux is about $1 \mathrm{erg} \mathrm{cm}^{-2} \mathrm{~s}^{-1}$. This may be compared to an energy flux of electrons of about $0.3 \mathrm{erg} \mathrm{cm}^{-2} \mathrm{~s}^{-1}$ deposited over the dayside hemisphere deduced from the $\mathrm{H}_{2}$ band emissions (Broadfoot et al., 1980). On the nightside, the flux of electrons capable of exciting $\mathrm{H}_{2}$ Lyman and Werner bands must be less than $0.3 \mathrm{erg} \mathrm{cm}^{-2} \mathrm{~s}^{-1}$, based on the absence of these emissions. The $L y \alpha$ nightglow implies an energy flux of about $0.04 \mathrm{ergs}^{-2} \mathrm{~s}^{-1}$, probably carried by low-energy electron or protons (McConnell et al., 1980b).

The increased $\mathrm{H}$ column abundance cannot be explained by dissociation by bombardment by heavy ions such as $\mathrm{S}$ or $\mathrm{O}$ ions from the Io plasma torus. Such ions need energies of about $100 \mathrm{MeV}$ to have a range equal to those of the energetic electrons penetrating the Jovian atmosphere as discussed above.

\section{CO-ROTATING MAGNETOSPHERIC CONVECTION}

We propose that the hydrogen bulge is created as a direct consequence of a co-rotating magnetospheric convection pattern that is driven by a longitudinal mass asymmetry in the Io torus. This asymmetry, which has been independently reported by Trauger et al. (1980), Pilcher and Morgan (1980), and Trafton (1980), can be accounted for by the magnetic-anomaly model in at least the following two ways:

(a) The magnetic-anomaly model is developed from the observation of the significantly nondipolar nature of the internal magnetic field of Jupiter (Smith et al., 1976; Acuña and Ness, 1976). The relevant magnetic-anomaly region lies in the northern hemisphere at surface levels in the area that includes part of the track of the foot of the Io flux tube and covering a longitude range $\lambda_{\text {III }}$ $(1965) \approx 170^{\circ}-235^{\circ}$; this region of anomalously weak surface magnetic fields is connected along magnetic field lines to the Io torus within the longitude range $\lambda_{\mathrm{III}} \approx 175^{\circ}-275^{\circ}$. The magneticanomaly related longitude ranges are referred to as the active sector (Vasyliunas, 1975; Dessler and Vasyliunas, 1979).

The area of the foot of a given magnetic tube of flux has a larger cross-sectional area within the active sector (where the surface field is relatively weaker) than at other longitudes. This effect is schematically illustrated in Fig. 6. Ionospheric plasma can escape more effectively from the anomaly region because of the relatively larger flux-tube foot (Dessler and Hill, 1975). The direct contribution of ionospheric plasma to an enhanced plasma density within the active sector is slight; however, as an ionizing source, it makes a primary contribution to creating the observed longitudinal asymmetry in the torus. Because of this ionospheric plasma, un-ionized gas escaping from Io is more likely to be ionized within the active sector.

(b) The magnetic mirror altitude for trapped energetic particles is a minimum, and the loss cone for precipitating auroral particles is a maximum within the active sector. Thus, the Jovian ionosphere is preferentially bombarded in this longitude range, which causes the conductivity of the ionosphere and the intensity of Birkeland currents to be locally enhanced (Dessler and Hill, 1979; Dessler, 1980). The Birkeland current density within the active sector reaches a magnitude such that current driven instabilities could be produced to further 


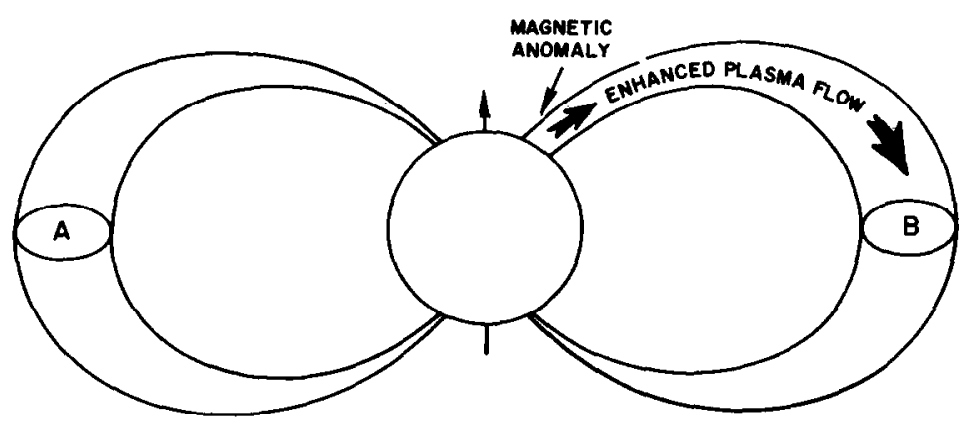

Fig. 6. Schematic Illustration of THE EFFECT OF a MAgNETIC ANOMALy ON THE FLOW OF IONOSPHERIC PLASMA INTO IO TORUS.

The two flux tubes $A$ and $B$ pass through the torus, and they both have the same cross-sectional area within the torus. However, flux tube $B$, which enters the magnetic anomaly region, has a larger foot at its northern end than either foot of flux tube $A$; the flow of ionospheric plasma from the magnetic anomaly into flux tube B is correspondingly larger than the related flow into A (after Dessler and Hill, 1975). While the direct contribution of this ionospheric plasma to the torus density is small, it provides an additional ionization source in the longitude range of the active sector. Un-ionized gas escaping from Io is more likely to become ionized within the active sector, thus resulting is a substantial longitudinal asymmetry in torus plasma density.

accelerate charged particles within the active sector (e.g., Smith and Goertz, 1978; Dessler and Hill, 1979; Dessler 1980). Such energized charged particles provide yet another ionizing source that cause gas escaping from Io to be ionized preferentially within the active sector, thus contributing to the longitudinal asymmetry in torus density described in (a). These energized particles would also produce the bright spot within the active sector that was discussed in Section 2. This particular mechanism has solid positive feedback: the strength of the Birkeland currents is determined by the longitudinal density gradients in the torus (Dessler, 1980), and these density gradients are established by ionizing bombardment by energized Birkelandcurrent particles.

Note that in (a) and (b) above, the magneticanomaly model has been embellished over the version being considered before the VOYAGER flybys by the inclusion of a relatively massive Io torus (cf., Dessler and Vasyliunas, 1979, and Vasyliunas and Dessler, 1980).

The Birkeland currents are part of a current loop as illustrated in Fig. 7 (Hill, 1980b). This current loop forms primarily in the active sector (Dessler, 1980) where the completion of the loop through the ionosphere produces an electric field $E=J \Sigma^{-1}$ where $J$ is the total current and $\Sigma$ is the height integrated conductivity of the ionosphere within the active sector. This electric field is always in the direction to cause the plasma in the torus to drift away from Jupiter; this same electric field propa- gates with varying intensity to other parts of the torus (and magnetosphere) to establish the convective flow illustrated in Fig. 8. This mechanism and the resulting convective flow pattern was first described by Vasyliunas (1978) and most recently, in some detail, by Hill et al. (1980).

The convective flow is outward, away from Jupiter, in the longitude interval in which the density of the Io torus is a maximum, which has been reported to be $\lambda_{\text {III }}=180^{\circ}$ (Trauger et al., 1980),

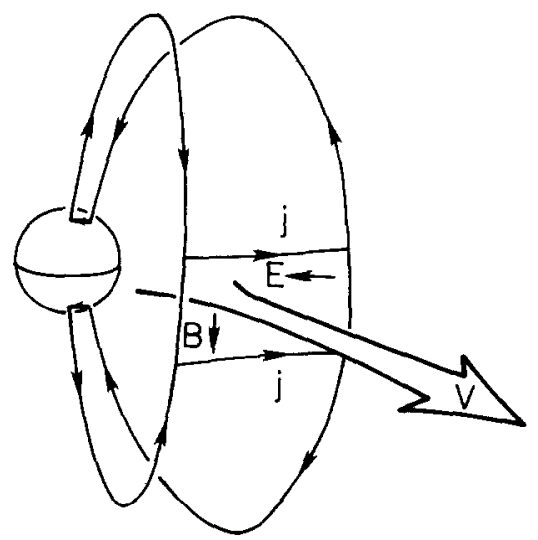

Fig. 7. BIRKELAND-CURRENT/RING-CURRENT/PEDERSENCURRENT-LOOP ESTABLISHED BY CENTRIFUGAL FORCE ACTING ON AN ELEMENT OF THE IO TORUS HAVING A LONGITUDINAL GRADIENT IN MASS DENSITY (AFTER HILL, 1980b).

The finite resistance in the ionosphere causes the Pedersen current to generate an electric field that initiates the co-rotating convection pattern described in the text. 


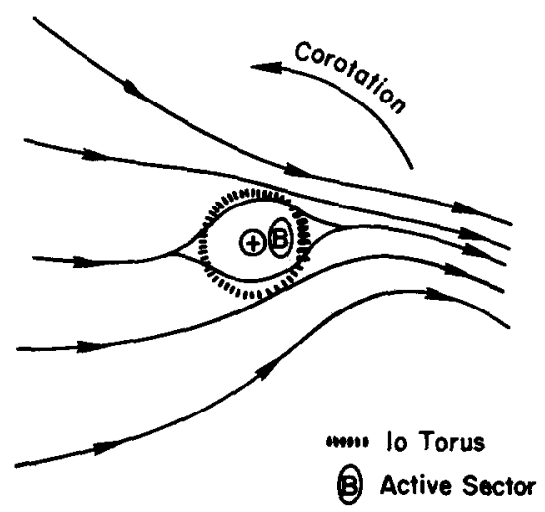

Fig. 8. THE DENSER PORTION OF THE Io PLASMA tORUS (THE PORTION OF THE TORUS IN THE ACTIVE SECTOR) IS FLUNG OUTWARD BY CENTRIFUGAI FORCE TO DRTVE A MAGNETOSPHERIC CONVECTION PATTERN THAT CO-ROTATES WTTH JUPITER (VASYLIUNAS, 1978; HILL, 1980a). SHOWN IS A SKETCH OF THE MAGNETOSPHERIC REGION OUT TO ABOUT $25 R_{J}$.

Plasma from the region of the active sector, labeled $B$ to correspond to flux tube B of Fig. 6, flows away from Jupiter to drive the convection pattern. This entire pattern is fixed relative to the active sector, and hence the convection pattern co-rotates with Jupiter even though the plasma itself might not co-rotate (Hill, 1979, 1980b). The outward convection occurs near $\lambda_{\mathrm{III}} \approx 250^{\circ}$, and the inward convection near $\lambda_{\mathrm{III}} \sim 90^{\circ}$. (These longitudes are uncertain by about $\pm 30^{\circ}$.) Hot plasma convected inward into the atmosphere causes the hydrogen bulge seen in Figs. 2 and 3 .

$\lambda_{\text {III }}=250^{\circ}$ (Pilcher and Morgan, 1980), and $\lambda_{\text {III }}=$ $260^{\circ}$ (Trafton, 1980 ). These maxima all fall within the active sector $\left(175^{\circ}<\lambda_{\text {III }}<275^{\circ}\right)$. The observations of Trafton (1980) are the most extensive, covering a four-year period, with that of Trauger et al. (1980) the least extensive, covering a three-day period. As illustrated in Fig. 8, plasma is convected inward, toward Jupiter, in a conjugate longitude interval roughly $180^{\circ}$ removed from the outward flow, which occurs at the longitude of the maximum in torus density. We thus expect the outward flow to usually be centered near $\lambda_{\mathrm{III}}=260^{\circ}$ (based on Trafton's (1980) observations), and, to zeroth order, thereforc, expect the most probable longitude for inward flow to be near $\lambda_{\text {III }}=$ $260^{\circ}-180^{\circ}=80^{\circ}$, with transient occurrences at smaller longitudes. Thus, according to this qualitative picture, the magnetic-anomaly influence on the co-rotating convection pattern produces an inward flow of magnetospheric plasma toward a central longitude that is consistent with the observed range of longitudes of the hydrogen bulge (i.e. $\lambda_{\text {III }}=80^{\circ}-$ $100^{\circ}$ ). The longitude of the hydrogen bulge moves in response to changes in the asymmetry in torus density. The longitude of the maximum torus density has been observed to vary over a range of $90^{\circ}$, but we do not think the hydrogen bulge will show this much motion. There are effects that tend to stabilize the convection pattern (see Hill et al, 1980 ), but bulge motion of $\pm 20^{\circ}$ in longitude should be commonly observed. Also, changes in the speed of the convective motion will cause the amplitude of the bulge to vary with time.

\section{JOVIGRA PHIC. STRUICTUIRE OF THF. HYDROGEN BULGE}

This torus-driven, co-rotating convection pattern brings hot plasma from the outer magnetosphere toward Jupiter at a nominal longitude $\lambda_{\text {III }} \sim 90^{\circ}$ (opposite the active sector). The inward moving flux tubes adiabatically compress the plasma they contain, thus energizing the plasma particles. These particles can bombard and heat Jupiter's upper atmosphere (Hunten and Dessler, 1977) as well as dissociate molecules to produce atomic hydrogen to sustain the hydrogen bulge. The particles execute an $\bar{E} \times \bar{B}$ drift toward Jupiter, the $E$ field having been generated, as outlined above, by the activesector portion of the torus. For particle energies below about $100 \mathrm{keV}$, gradient and curvature drift motions can be neglected. Because the convection electric field pattern co-rotates with Jupiter, the $\bar{E} \times \bar{B}$ drift can carry particles all the way down to the ionosphere. This is in contrast to plasma motion in the Earth's magnetosphere in which Earthward convective flow from the magnetotail is deffected relatively far above the ionosphere (usually 8$10 R_{E}$ ) by a combination of gradient and curvature drifts plus a co-rotational electric field pattern that is superposed on the standard two-cell pattern that remains fixed relative to the solar wind.

The Jupiterward drift is centered about the particle drift equator. This equator is defined as the locus of energetic particles at ionospheric height having $90^{\circ}$ pitch angle (i.e. particles whose north and south mirror points are coincident). The position of this equator, as shown in Figs. 2 and 3, was taken from Fig. 5 of Acuña and Ness (1976). Energetic particle populations drifting either toward or around Jupiter tend to be centered around this line, hence the designation "particle drift equator". It is clear from inspection of Figs 2 and 3 that the isophotes in the region of the hydrogen bulge $\left(170^{\circ}>\lambda_{\mathrm{mI}}>0^{\circ}\right)$ follow the particle drift equator and not the spin equator. At other longitudes the isophotes continue to show a weak tendency to be organized relative to the particle drift equator (see Fig. 2), although not as strongly 
as in the hydrogen bulge region. We interpret this as indicating that some fraction of the atomic hydrogen is created at virtually all longitudes by energetic particle bombardment.

More evidence that the hydrogen bulge is caused by inward drifting plasma is contained in scan 1 of Fig. 4. This north-south scan, as indicated in Fig. $2 a$, passed through the hydrogen bulge at a longitude near its center. The peak is bifurcated with one maximum at $\phi=22^{\circ}$ and the other maximum at $\phi=-2^{\circ}$. ( $\phi$ is the Jovigraphic latitude angle with positive values for north latitudes.) Such a bifurcation is expected as the hot plasma is convected toward Jupiter. Because of pitch-angle scattering, the flux tubes are emptied principally by particles being scattered into the local loss cone and precipitated into the atmosphere. Particles are thus preferentially lost out of the ends of a tube of flux to produce the atomic hydrogen that sustains the bulge; particles can reach the drift equator only by being carried in by the convective motion. We therefore expect a relative minimum at the particle drift equator.

The latitudinal separation of the two peaks $\left(24^{\circ}\right)$ is also about what is expected. In a dipole approximation, the precipitating particles that produce the peaks are on an $L$-shell, $L=1 / \cos ^{2} \psi$ where $\psi$ is the magnetic latitude. For $\psi=12^{\circ}, L=1.045$, which corresponds to a flux tube whose equatorial altitude is $3.2 \times 10^{3} \mathrm{~km}$ above the altitude of particle precipitation at the ends of the flux tube. This is approximately the altitude where significant atmospheric interaction is expected to rapidly empty the flux tube so that relatively few particles are available for further inward convection; the production of atomic hydrogen, therefore, becomes relatively weaker as the remaining particles continue to move inward. The expected bifurcated structure is not as evident in scan 2 (Fig. 4), probably because of non-magnetic-anomaly (i.e. solar) effects that dominate at larger longitudes. However, faint maxima can be seen (ignoring the single point at $\phi=7^{\circ}$ ) at $\phi=14^{\circ}$ and $\phi=-13^{\circ}$.

\section{DISCUSGION}

The bulge structure is unique to Jupiter among the planets studied to date; an understanding of the underlying cause is likely to lead to a fundamental advance in our understanding of Jupiter's environment. Even with the success of the magneticanomaly model in accounting for the location and character of the Lyo bulge, several characteristics of the emissions from Jupiter's atmosphere remain unexplained. Perhaps the biggest puzzles result from the disappearance (down to less than $10 \%$ of their dayside intensity) of the $\mathrm{H}_{2}$ bands on the nightside, implying a day-night asymmetry in electron precipitation. The magnetic-anomaly model does not offer a day-night asymmetry, indicating that other precipitation processes are probably present. This conclusion is consistent with the fact that the $\mathrm{H}_{2}$ bands show no longitudinal structure comparable to the Ly $\alpha$ bulge, a further indication that the $\mathrm{H}_{2}$ band excitation and the $L y \alpha$ bulge are in some sense separate phenomena. Thus it seems likely that the best explanation of the characteristics of Jupiter's disc emission includes two sources of electron precipitation. The first excites the $\mathbf{H}_{2}$ band emission and has a strong day-to-night variation. The second source probably results from the co-rotating plasma convection as described above and provides an increased $\mathrm{H}$ column abundance at certain longitudes, thereby accounting for the $L y \alpha$ intensity bulge. Whether this same effect is responsible for the precipitation asymmetry detected on the nightside (McConnell et al., 1980b) is yet uncertain.

Convection within the Earth's magnetosphere is usually detected through its physical consequences; the overall convective motions are seldom seen directly. Thus it is with Jupiter; the hydrogen bulge is explained as a direct consequence of a corotating convection pattern that is theoretically expected by the magnetic-anomaly model. This is the first experimental observation of a co-rotating convection effect. We are not aware of any alternate explanations for the hydrogen bulge, although the absence of a competing explanation does not necessarily make the one offered here correct. Other corroborating evidence is required before we can claim that co-rotating magnetospheric convection has been established for Jupiter. Differences between co-rotating convection and sun-fixed convection (as in the Earth's magnetosphere) are so striking that it is likely that additional tests can be devised in which experimental findings can be compared with quantitative consequences of the magnetic-anomaly model and its attendant corotating convection.

Acknowledgements-We arc grateful to J. C. McConncll, J. E. P. Connerney, T. W. Hill, and R. A. Wolf for useful suggestions. AJD acknowledges support in part by the Division of Atmospheric Sciences, National Science Foundation under Grant ATM78-21767 and the Solar Terrestrial Division, National Aeronautics and Space Administration under Grant NGR 44-006-137. BRS acknowledges support by the Jet Propulsion Laboratory, California Institute of Technology under NASA contract NAS 7-100, and by the Planetary Sciences Discipline of 
NASA's Office of Space Sciences. SKA acknowledges support from a research grant from the NASA Planetary Atmospheres Program.

\section{REFERENCES}

Atreya, S. K., Yung, Y. L., Donahue, T. M. and Barker, E. S. (1977). Search for Jovian auroral hot spots. Astrophys. J. 218, L83.

Acuña, M. H. and Ness, N. F. (1976). The main magnetic field of Jupiter, in Magnetospheric Particles and Fields (Ed. B. M. McCormac). Reidel. Dordrecht.

Broadfoot, A. L., Sandel, B. R., Shemansky, D. E., Atreya, S. K., Donahue, T. M., Moos, H. W., Bertaux, J. L., Blamont, J. E., Ajello, J. M., Strobel, D. F., McConnell, J. C., Dalgarno, A., Goody, R., McElroy, M. B. and Yung, Y. L. (1977). Ultraviolet spectrometer experiment for the Voyager mission. Space Sci. Rev. 21, 183.

Broadfoot, A. L., Sandel, B. R., Shemansky, D. E., McConnell, J. C., Smith, G. R., Holberg, J. B., Atreya, S. K., Donahue, T. M., Strobel, D. F. and Bertaux, J. L. (1980). Overview of the Voyager ultraviolet spectrometry results through Jupiter encounter. J. geophys. Res. (in press).

Clarke, J. T., Weaver, H. A., Feldman, P. D., Moos, H. W., Fastie, W. G. and Opal, C. B. (1980). Spatial imaging of Hydrogen Lyman $\alpha$ emission from Jupiter. Astrophys. J. 240, 696.

Dessler, A. J. (1980). Corotating Birkeland currents in Jupiter's magnetosphere: an Io plasma-torus source. Planet. Space Sci., 28, 781.

Dessler, A. J. and Chamberlain, J. W. (1979). Jovian longitudinal asymmetry in Io-related and Europarelated auroral hot spots. Astrophys. J. 230, 974.

Dessler, A. J. and Hill, T. W. (1975). High-order magnetic multipoles as a source of gross asymmetry in the distant Jovian magnetosphere. Geophys. Res. Lett. 567.

Dessler, A. J. and Hill, T. W. (1979). Jovian longitudinal control of Io-related radio emission. Astrophys. J. 227, 664.

Dessler, A. J. and Vasyliunas, V. M. (1979). The magnetic anomaly model of the Jovian magnetosphere: predictions for Voyager. Geophys. Res. Lett. 6, 37.

Festou, M. C., Atreya, S. K., Donahue, T. M., Sandel, B. R., Shemansky, D. E. and Broadfoot, A. L. (1980). Composition and thermal profiles of the Jovian upper atmosphere determined by the Voyager ultraviolet stellar occultation experiment. Submitted to J. geophys. Res.

Goldreich, P. and Lynden-Bell, D. (1969). Io, a Jovian unipolar inductor, Astrophys. J. 156, 59.
Hill, T. W. (1979). Inertial limit on corotation. J. geophys. Res. 84, 6554.

Hill, T. W: (1980a). Torus convection, Conference on the Physics of the Jovian Magnetosphere, February 27-29, Rice Univ., Houston.

Hill, T. W. (1980b). The Jovian magnetosphere: a postVoyager view, $E \oplus S$, Trans. AGU (in press).

Hill, T. W., Dessler, A. J. and Maher, L. J. (1980). Co-rotating magnetospheric convection. Submitted to $J$. geophys. Res.

Hunten, D. M. and Dessler, A. J. (1977). Soft electrons as a possible heat source for Jupiter's thermosphere. Planet. Space Sci. 25, 817.

McConnell, J. C., Sandel, B. R. and Broadfoot, A. L. (1980a). Voyager UV spectrometer observations of He $584 \AA$ dayglow at Jupiter. Planet. Space Sci. (in press).

McConnell, J. C., Sandel, B. R. and Broadfoot, A. L. (1980b). Airglow from Jupiter's night side and crescent: Ultraviolet spectrometer observations from Voyager 2. Icarus 43, 128.

Pilcher, C. B. and Morgan, J. S. (1980). The distribution of [SII] emission around Jupiter. Astrophys. J. 238, 375.

Sandel, B. R., Broadfoot, A. L. and Strobel, D. F. (1980). Discovery of a longitudinal asymmetry in the Hydrogen Lyman-alpha brightness of Jupiter. Geophys. Res. Lett. 7,5 .

Smith, E. J., Davis, L., Jr. and Jones, D. E. (1976). Jupiter's magnetic field and magnetosphere, in Jupiter (Ed. T. Gehrels) pp. 788-829. Univ. of Arizona Press, Tucson.

Smith, R. A. and Goertz, C. K. (1978). On the modulation of the Jovian decametric radiation by Io: 1 . Acceleration of charged particles, J. geophys. Res. 83, 2617.

Strobel, D. F. (1979). The ionosphere of the major planets. Rev. Geophys. Space Phys. 17, 1913.

Trafton, L. (1980). Jovian SII Torus: Its longitudinal asymmetry. Icarus, 42, 111.

Trauger, J. T., Münch, G. and Roesler, F. L. (1980). A study of the Jovian [SII] nebula at high spectral resolution. Astrophys. J. 236, 1035.

Vasyliunas, V. M. (1975). Modulation of Jovian interplanetary electrons and the longitude variation of decametric emission. Geophys. Res. Lett. 2, 87.

Vasyliunas, V. M. (1978). A mechanism for plasma convection in the inner Jovian magnetosphere, Cospar Program/Abstracts, p. 66, Innsbruck, Austria.

Vasyliunas, V. M. and Dessler, A. J. (1980). The magnetic-anomaly model of the Jovian magnetosphere: A post-Voyager assessment. J. geophys. Res., (in press).

Yung, Y. L. and Strobel, D. F. (1980). Hydrogen photochemistry and Lyman-alpha albedo of Jupiter. Astrophys. J. 239, 395. 